\title{
Imisopasem Manganese
}

National Cancer Institute

\section{Source}

National Cancer Institute. Imisopasem Manganese. NCI Thesaurus. Code C111684.

A mang anese-based non-peptidyl mimetic of the human mitochondrial mang anese superoxide dismutase (MnSOD), with potential antioxidant and chemo/radioprotective activities. Upon administration, imisopasem mang anese mimics the activity of MnSOD and scavenges reactive oxygen species (ROS), such as superoxide anion, which prevents oxygen free radical damage to macromolecules such as DNA. This reduces ROSmediated lipid peroxidation, prevents apoptosis and protects against oxygen free radicalinduced toxicity in normal tissues. 